prognosis (participant's own perception). $66.7 \%$ of self-perceived high-activity RA by patients exceeded the physician's impression; and $31.03 \%$ of the intermediateactivity. The degree of knowledge and the importance given to certain aspects of the disease in relation to the poor prognosis ranged from 8 to $9 / 10$ in the different items analyzed. The awareness of their seropositivity against FR and ACPA was $77 \%$ and $17 \%$, respectively. $63 \%$ consider the physical aspect more disabling, but $52 \%$ gave more value to feeling good emotionally. The mean treatment score for physical aspects was 7.07 (SD 2.52), whereas for emotional aspects was 3.39 (SD 2.57). $51.9 \%$ of self-considered poor prognosis patients believed that biological treatment was delayed and $50 \%$ related that to poorer outcome. $97 \%$ wanted to participate in the doctor's decisions, but $38 \%$ declared they had no choice. There were no statistically significant differences between the prognostic groups.

Conclusions: More than half of the patients self-reported poor prognosis. Twothirds estimated poorer health status than physicians if their RA was of high activity; $1 / 3$ if it was intermediate. Patients considered all assessed aspects as determinants of poor prognosis $(m>8)$. The degree of knowledge of their condition was high, being lower for joint damage and activity and prognostic markers. The beginning of treatment with biologicals was perceived as delayed in more than half of those who declared themselves with poor prognosis and half of them related it to a worse evolution. A high percentage of patients showed low satisfaction with the emotional attention received. Most claim to be more involved in medical decisions, although more than $1 / 3$ does not seem to be able to do so.

Acknowledgements: Coordinadora Nacional de Artritis (ConArtritis)

Disclosure of Interest: None declared

DOI: 10.1136/annrheumdis-2017-eular.5336

\section{AB1193 DEVELOPING THE KOREAN EDUCATIONAL NEEDS ASSESSMENT TOOL (KOREAN-ENAT) IN RHEUMATOID ARTHRITIS: A CROSS-CULTURAL VALIDATION USING RASCH ANALYSIS}

Y.-K. Sung ${ }^{1}$, D. Kim ${ }^{1}$, S.J. Cha ${ }^{1}$, D. Yoo ${ }^{1}$, S.-H. Kim ${ }^{2}$, M. Ndosi ${ }^{3}$, S.-K. Cho ${ }^{1}$. ${ }^{1}$ Department of Rheumatology, Hanyang University Hospital for Rheumatic Diseases, Seoul; ${ }^{2}$ Department of Measurement and Evaluation of Physical Education, Chungbuk National University, Cheongju, Korea, Republic Of; ${ }^{3}$ Centre for Health and Clinical Research, University of the West of England, Bristol, United Kingdom

Background: The Educational Needs Assessment Tool (ENAT) is a 39-item patient-completed questionnaire designed to help patients identify and prioritize their educational needs. It was originally developed in the UK and validated in 7 rheumatic diseases including rheumatoid arthritis (RA). ${ }^{1}$

Objectives: This study aimed to undertake cross-cultural adaptation and validation of the ENAT in RA for use in Korea.

Methods: The study involved two main phases: (1) Cross-cultural adaptation of the ENAT from English into Korean and (2) validation of the Korean-ENAT. The first phase followed an established process of cross-cultural adaptation of self-report measures. ${ }^{2}$ For the second phase, patients with RA completed the Korean-ENAT at the outpatient clinic of a university hospital and Rasch measurement computer program, WINSTEPS, was used to analyze the data. Fit to the model was determined by the observed data Infit and Outfit statistics ( $\geq 0.50$ and $\leq 1.50$ ); where a value of 1.00 suggests a perfect fit to the model expectations. The unidimensionality of the scale was determined by item (and person) separation index $>2.00$ and reliability $>0.80$

Results: An adequate conceptual equivalence was achieved following the adaptation process. A total of 123 patients completed the Korean-ENAT. Their mean \pm SD age was $46.7 \pm 12.3$, disease duration $53.7 \pm 71.2$ months and the majority (81.3\%) were female. Thirty-five of the 39 items displayed good fit to the model. The 4 items deviating from the model had Infit and Outfit $>1.50$. The item separation index (5.26) and item reliability index (0.97) provided evidence

Table 1. Fit statistics for the Korean ENAT subscales

\begin{tabular}{|c|c|c|c|c|c|}
\hline \multirow[b]{2}{*}{ Subscales } & \multicolumn{2}{|c|}{ Infit } & \multicolumn{2}{|c|}{ Outfit } & \multirow{2}{*}{$\begin{array}{c}\text { Point-biserical correlation } \\
\text { PTMEA CORR. }\end{array}$} \\
\hline & MNSQ & $\overline{\text { ZSTD }}$ & MNSQ & ZSTD & \\
\hline Pain & 1.31 & 2.30 & 1.40 & 2.80 & 0.75 \\
\hline Movement & 1.00 & 0.10 & 1.01 & 0.10 & 0.85 \\
\hline Feelings & 0.77 & -1.90 & 0.80 & -1.60 & 0.84 \\
\hline Disease & 1.27 & 2.00 & 1.15 & 1.00 & 0.84 \\
\hline Treatments & 1.30 & 2.20 & 1.21 & 1.40 & 0.85 \\
\hline Self-help & 1.05 & 0.50 & 1.08 & 0.70 & 0.84 \\
\hline Support & 0.75 & -2.10 & 0.75 & -2.10 & 0.83 \\
\hline
\end{tabular}

$\overline{\mathrm{MNSQ}}=$ mean-square; ZSTD $=$ z-standardized; MNSQ between $\geq 0.50$ and $\leq 1.50$ for model fit. for good reliability of items. All the 7 domains of the Korean-ENAT were found to fit the Rasch model. The internal consistency of the Korean-ENAT was high and unidimensionality was confirmed (Person separation index $=3.41$ reliability index $=0.92$; item separation index $=16.82$ and reliability index $=1.00$ ).

Conclusions: Using a standard process in cross-cultural adaptation, the ENAT was adapted into Korean and Rasch analysis confirmed that the construct validity, reliability, and unidimensionality of the Korean-ENAT. The Korean-ENAT provides valid and reliable estimates of educational needs of people with RA in Korea. References:

[1] Ndosi $\mathrm{M}$, et al. Validation of the educational needs assessment tool as a generic instrument for rheumatic diseases in seven European countries. Ann Rheum Dis 2014; 73: 2122-9.

[2] Beaton DE, et al. Guidelines for the process of cross-cultural adaptation of self-report measures. Spine 2000; 25: 3186-91.

Disclosure of Interest: None declared

DOI: 10.1136/annrheumdis-2017-eular.4549

\section{AB1194 ANTINUCLEAR ANTIBODIES ALGORITHM FOR THE PRIMARY HEALTH CARE PHYSICIANS IN CYPRUS}

V. Scoutellas ${ }^{1}$, G. Mitis ${ }^{2}$, G. Achniotou ${ }^{3} .{ }^{1}$ Rheumatology Department;

${ }^{2}$ Immunology Department, Nicosia General Hospital; ${ }^{3}$ Health Insurance Organization, Nicosia, Cyprus

Background: due to our daily clinical and laboratory practice, we realized that primary care physicians of Cyprus (public \& private sector), as well as other medical specialties, are not familiar with the clinical significance of autoantibodies used to diagnose autoimmune rheumatological diseases. This resulted to an increasing number of orders for autoantibodies, which were irrelevant to the person's condition, leading to a high expenditure for tests not clinically needed, whereas the laboratory was not able to respond to the demand with the available resources (human, hardware, economic).

Objectives: (a) to persuade, through education, all the primary care physicians of Cyprus to order autoantibodies up to the point of the primary healthcare level and (b) to minimize the number of unneeded orders, as well as the expenditure, and save resources. To accomplish this target, we created an algorithm for ordering antinuclear antibodies (ANA). Mainly the primary care physicians should use the algorithm as a protocol.

Methods: we searched the literature to find (a) existing algorithms for ordering the ANA, as well as other autoantibodies that should be performed if ANA is found positive and (b) the relevant scientific evidence to guide primary care physicians while ordering ANA. In addition, we planned educational lectures for all the primary care physicians (public \& private sector). Our scope was to develop an algorithm that guides the primary care physician to order antinuclear antibodies only if the person's symptoms are relevant to an autoimmune rheumatological disease.

Results: we found algorithms from different sources (university clinics, scientific societies [medical and laboratory], commercial companies producing autoantibody kits). Most of the algorithms found were focusing on the suspected disease, for which the relevant autoantibodies should be ordered. We established an algorithm targeting the primary care physicians: they should only order antinuclear antibodies if an autoimmune rheumatology disease is suspected; if ANA is negative, then an autoimmune disease does not exist and the test should never be repeated (unless there is an important reason); if ANA is positive, then the person should be sent for a consultation to a rheumatologist, who is responsible to take the medical history, examine clinically the person and decide if there is a need or not to order for any other autoantibodies, if an autoimmune rheumatology condition is still suspected. The developed algorithm was communicated (year 2014) to the primary care physicians through lectures in small teams (it was repeated in seven different teams, and captured almost all). The Cyprus Rheumatology Society acknowledged the algorithm, whereas the Health Insurance Organization, with the Ministry of Health, organized the educational part.

Conclusions: the development and establishment (years 2015-2016) of the algorithm for the ordering of antinuclear antibodies by the primary care physicians, changed their attitude towards ordering nowadays with more scientific and evidenced based criteria. This was seen on the lower numbers of orders of antinuclear antibodies within these two years.

References:

[1] the developed algorithm, with the associated text and lecture (all in pdf format), can be viewed (greek language only) and/or downloaded from http://www.hio.org.cy/gr/kko_ergkon_eksetaseon_pfy.html.

Disclosure of Interest: None declared

DOI: 10.1136/annrheumdis-2017-eular.2033 